\title{
Bradyarrhythmias, temporary and permanent pacing
}

\author{
Vineet Kaushik, MD; Angel R. Leon, MD; James S. Forrester Jr, MD; Richard G. Trohman, MD
}

Bradycardia is common in critical care units. It may be transient, asymptomatic and of little consequence, or life-threatening. Bradycardia may result from abnormalities of the sinus node, atrioventricular node, or the His-Purkinje system. It may also be precipitated by drug effects or enhanced vagal tone. Proper

\begin{abstract}
diagnosis is pivotal to determining prognosis and management. Temporary and permanent pacing is now readily available, markedly improving the morbidity and mortality associated with bradyarrhythmias. (Crit Care Med 2000; 28[Suppl.]:N121-N128)
\end{abstract}

KeY WoRDS: sinus node dysfunction, atrioventricular block, pacing

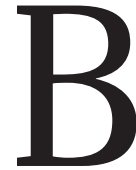

radyarrhythmias are frequently encountered in the critical care setting. They can present as incidental findings or potentially life-threatening arrhythmias. Proper identification is key to determining prognosis and management. Before the advent of pacemakers, the prognosis of certain bradyarrhythmias was dismal. Fortunately, temporary and permanent pacing is now readily available, markedly improving morbidity and mortality associated with bradyarrhythmias.

Basic Anatomy and Physiology of the Conduction System. The normal heartbeat arises from the sinoatrial (SA) node, located in the right atrium near its junction with the superior vena cava. The SA node serves as the main pacemaker of the heart under normal conditions. The blood supply to the SA node is predominantly via the SA nodal artery, a branch of the right coronary artery in $\sim 60 \%$ of people and left circumflex coronary artery in $40 \%$ of people. The electrical impulse travels from the SA node in a radial fashion depolarizing both atria before reaching the atrioventricular $(\mathrm{AV})$ node.

The AV node is located posteriorly in the right atrium adjacent to the tricuspid annulus. The anatomy of the AV junction is complex and is comprised of three regions: the transitional zone, compact $\mathrm{AV}$

From the Carlyle Frasier Heart Center at Crawford Long Hospital, Division of Cardiology, Emory University School of Medicine (Drs. Kaushik, Leon), and the Division of Cardiovascular Disease and Critical Care Medicine, Rush-Presbyterian St. Luke's Medical Center (Drs. Forrester, Trohman).

Address requests for reprints to: Angel R. Leon, $M D$, The Carlyle Fraser Heart Center at Crawford Long Hospital, 550 Peachtree Street, NE, Atlanta, GA 30365.

Copyright $\odot 2000$ by Lippincott Williams \& Wilkins node, and the penetrating AV (His) bundle. The blood supply to the AV node is via the AV nodal artery, which arises from the distal right coronary artery in $90 \%$ of people and left circumflex coronary artery in $10 \%$ of people. As electrical impulses travel through the AV node, a normal delay occurs (which accounts for much of the P-R interval) and serves two primary purposes. First, delay within the AV node allows time for atrial contraction to effectively contribute to ventricular filling. Second, the delay limits very rapid transmission of atrial impulses to the ventricles in cases of supraventricular tachycardia. Indeed, incremental increases in the rate of atrial pacing in the Electrophysiology lab results in progressive AV nodal conduction delay before block occurs (1). This manifests itself on the surface electrocardiogram (ECG) by progressive prolongation of the P-R interval before AV block.

The cardiac impulse then travels through the His bundle to the Purkinje system and the ventricular myocardium, allowing ventricular depolarization. Block at the level of the His-Purkinje system generally occurs abruptly (with no progressive AV delay) without P-R interval prolongation.

Both the SA node and AV node are richly innervated by the autonomic nervous system. The relative balance between the parasympathetic and sympathetic nervous system input accounts for the resting (baseline) autonomic tone. Studies measuring effects of autonomic blockade on the conduction system have shown that, at rest, parasympathetic activity predominates at the SA node. Parasympathetic and sympathetic activity are nearly equally balanced in the AV node (2). As we shall see, an increase in para- sympathetic output (increased vagal tone) can result in clinically significant bradyarrhythmias.

\section{Sinus Node Dysfunction}

Bradycardia resulting from sinus node dysfunction can be divided into two broad categories-failure of impulse generation or failure of impulse conduction. Failure of impulse generation refers to an abnormal ability of the SA node to generate an impulse (abnormal automaticity). Failure of conduction occurs when an appropriately generated SA impulse fails to depolarize the atria because of exit block. Common electrocardiographic manifestations of sinus node dysfunction include inappropriate sinus bradycardia, sinus pauses, sinus exit block, and chronic atrial fibrillation with a slow ventricular response, and the bradycardia-tachycardia syndrome.

Sinus Bradycardia. The normal heart rate is arbitrarily defined as ranging between 60 and 100 beats/min (bpm). Sinus bradycardia (heart rate $<60 \mathrm{bpm}$ ) does not equate with sinus node dysfunction. In fact, heart rates $<40 \mathrm{bpm}$ can be common and asymptomatic in people at rest, particularly in well-trained athletes (3). Sinus bradycardia is considered "inappropriate" and clinically pathologic when symptomatic or when associated with a failure to appropriately increase heart rate during activity or vigorous exercise.

Sinus Pause or Arrest. Sinus pause or sinus arrest occurs when the SA node transiently fails to exhibit normal automaticity. The ECG demonstrates a pause without P waves (Fig. 1). The pause will not be a multiple of the preceding P-P interval. Pauses $>2$ secs and $<3$ secs are seen in $11 \%$ of healthy asymptomatic individuals, whereas pauses $>3$ secs during 


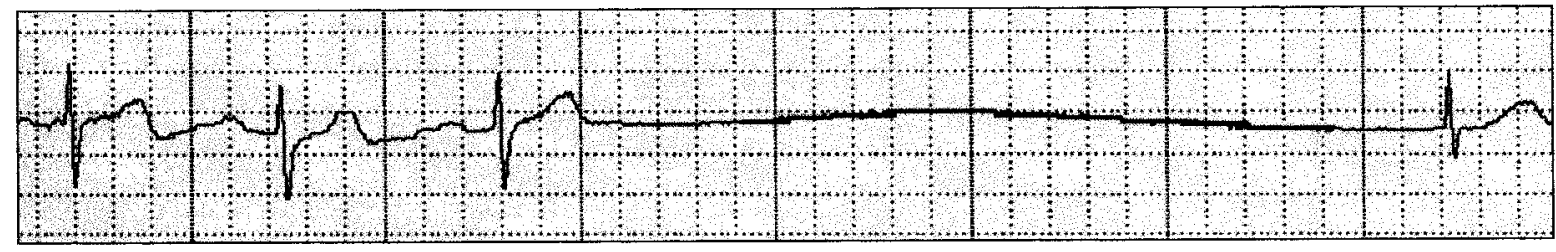

Figure 1. In this patient with sinus arrest, a $5.64-\mathrm{sec}$ pause is terminated by a junctional escape beat.

waking hours are usually because of sinus node dysfunction.

Sinus Exit Block. Sinus exit block also results in a pause. In this case, the SA node fires; however, the impulse is delayed or fails to propagate beyond the sinus node, resulting in failure of atrial depolarization. The ECG again shows a pause without $\mathrm{P}$ waves. Type I seconddegree SA exit block demonstrates progressive P-P interval shortening before the pause (duration is less than $2 \mathrm{P}-\mathrm{P}$ cycles). In Type II second-degree sinoatrial exit block, the pause duration is theoretically an exact multiple of the previous P-P interval as the SA node continues to fire at its own intrinsic rate. SA node firing is not recorded on the surface ECG (Fig. 2).

Bradycardia-Tachycardia Syndrome. Bradycardia-tachycardia syndrome refers to the presence of sinus node dysfunction with both bradycardia and tachycardia (usually demonstrated as paroxysmal atrial fibrillation on surface ECG). Holter monitor studies have demonstrated that clinical symptoms of presyncope or syncope usually arise from the long pauses that accompany termination of the tachycardia (Fig. 3).

\section{Conduction Disturbances Involving the Atrioventricular Node/His Bundle}

Abnormalities of the AV node and His bundle are primarily manifested as disturbances in impulse conduction. Clinically, it is important to distinguish between block within the AV node and block within or below the His bundle (infranodal AV block), as both prognosis and appropriate treatment depend on this distinction. The surface ECG can be valuable (but not infallible) in providing clues to help assist in making this differentiation. First, as discussed earlier, prolongation of the P-R interval before block (Wenckebach pattern) is strongly suggestive of AV nodal block, whereas sudden block without prolongation of the P-R interval is very suggestive of infranodal block.

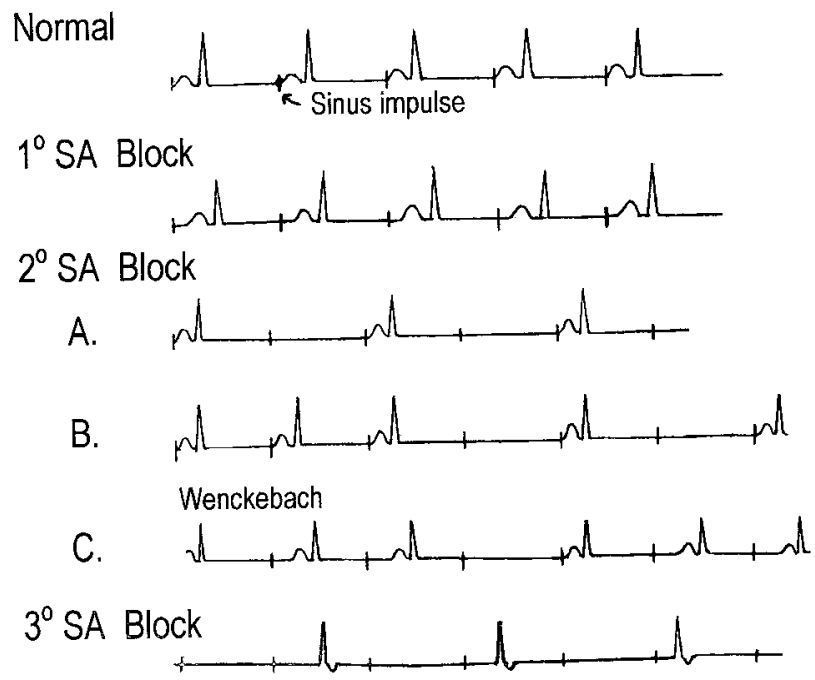

Figure 2. First-degree sinoatrial exit block is characterized by intranodal delay and is not visible on surface electrocardiogram. $C$, in sinoatrial Wenckebach P-P shortening precedes a pause. $B$, in Type II second-degree sinoatrial block, the pause is a multiple of the intrinsic P-P interval. Adapted with permission from Chou T, Knilans TK (Eds): Electrocardiography in Clinical Practice: Adult and Pediatric. Philadelphia, WB Saunders, 1996, p 330.

Second, the escape rate in the setting of complete AV block is higher ( $40-60 \mathrm{bpm})$ in AV nodal block, as the escape pacemaker is usually in the His/proximal Purkinje system. Infranodal block results in a less reliable, more distal (often ventricular) escape rhythm (heart rate $<40 \mathrm{bpm}$ ). Third, the width of the QRS complex is helpful for similar reasons. The QRS duration in the setting of $\mathrm{AV}$ nodal block is generally relatively narrow ( $<120$ msecs) whereas the QRS duration in the infranodal block is relatively wide.

First-Degree AV Block. In first-degree AV block, each atrial impulse is successfully conducted to the ventricle but with a delay. Thus, each $P$ wave is followed by a QRS but with prolongation in the P-R interval. A P-R interval $\geq 210$ msecs is diagnostic. This delay can theoretically occur anywhere along the conduction system; however, it occurs predominantly within the AV node or above. When the QRS complex is narrow (most common), the level of conduction delay is within the $\mathrm{AV}$ node in $>90 \%$ of cases (1). However, first-degree AV block with a bundle- branch block/wide QRS may represent infranodal conduction delay in up to $45 \%$ of patients (1).

Second-Degree AV Block-Mobitz Type I (Wenckebach). In all types of seconddegree heart block, only some atrial impulses reach the ventricle. It follows that the surface ECG demonstrates some P waves that are not followed by a QRS complex. In typical Mobitz type I block, there is progressive prolongation of the $\mathrm{P}-\mathrm{R}$ interval with shortening of the R-R intervals before the blocked cycle. Atypially, there is an associated shortening of the P-R interval in the first conducted cycle after the block (Fig. 4). Again, Wenckebach block is almost always a result of block in the AV node particularly when associated with a narrow QRS complex.

Second-Degree AV Block-Mobitz Type II. Like second-degree-Type I block, some $\mathrm{P}$ waves are not conducted to the ventricles and fail to produce a QRS. However, unlike Type I block, Type II block occurs without prolongation in the cal R-R responses are common. Addition- 


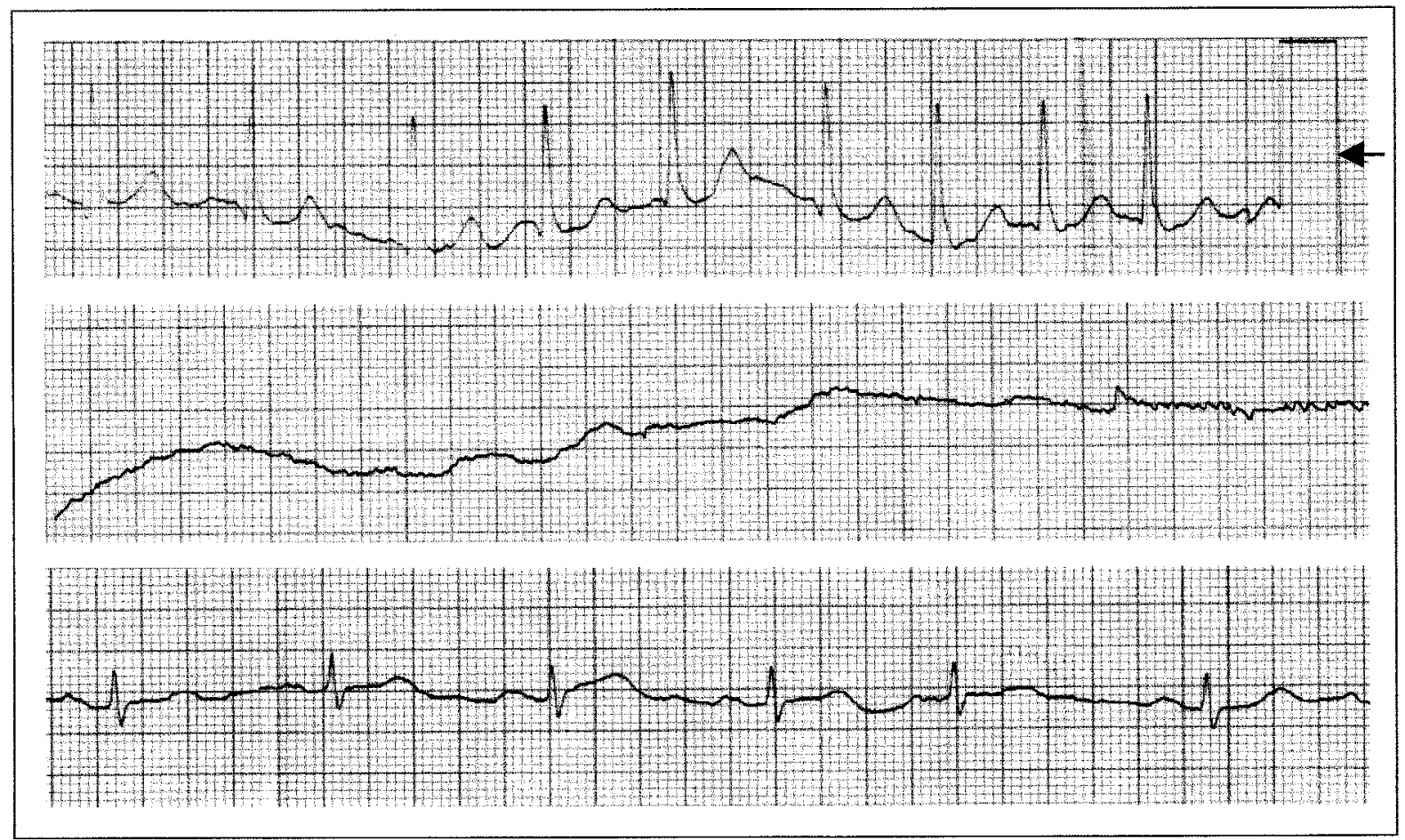

Figure 3. In this patient with bradycardia-tachycardia syndrome, the top tracing demonstrates atrial fibrillation with a rapid ventricular response. After direct current cardioversion (arrow), there is atrial and ventricular asystole (middle tracing) lasting $\sim 6$ secs before resumption of sinus rhythm (bottom tracing).

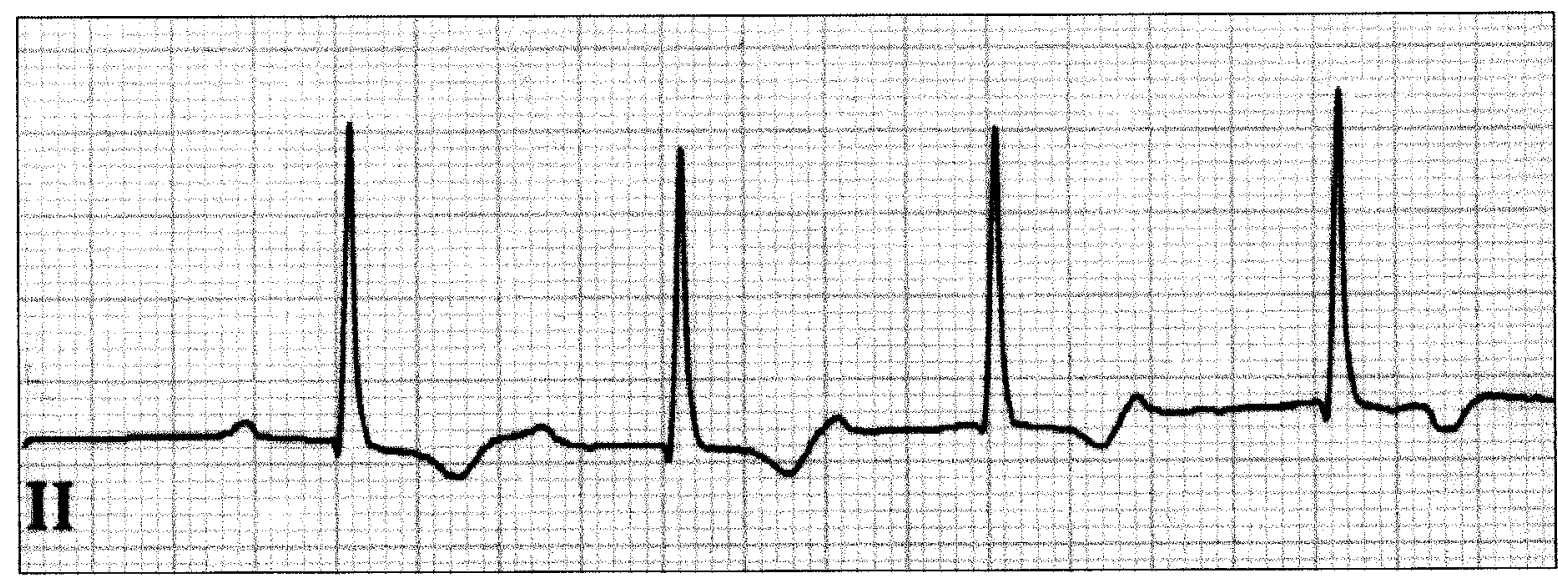

Figure 4. Mobitz Type I (Wenckebach) atrioventricular block is characterized by gradual P-R interval prolongation. The fifth P wave (seen distorting the T wave after the fourth QRS complex) was not conducted. II, standard ECG lead.

preceding P-R intervals. There is no change in the first conducted P-R interval after the blocked $\mathrm{P}$ wave (Fig. 5). The level of block is usually infranodal, and thus potentially very dangerous because electrical activation of the ventricles may depend on more unstable and unreliable distal automaticity.

2:1 AV Block. In this special case, every other $\mathrm{P}$ wave is followed by a QRS, making it impossible to tell if P-R prolongs before the nonconducted $\mathrm{P}$ waves (Fig. 6). In addition to the QRS duration, certain maneuvers based on the normal conduction properties of the AV node and His-Purkinje System can be performed in a critical care unit to help distinguish the site of block (Table 1).

Third-Degree AV Block. Complete heart block occurs when no atrial impulses are conducted to the ventricles resulting in electrocardiographic findings of complete atrioventricular disassociation (Fig. 7). The level of block can be either in the AV node or infranodal; however, acquired causes (which predomi- nate in a critical care setting) are often infranodal and potentially life-threatening. A common exception to this can occur in the setting of an acute inferior myocardial infarction (MI) and is discussed later. Digitalis toxicity is a common cause of reversible AV block. Again, the width and rate of the QRS escape rhythm are very helpful in determining the site of block (4).

Neurally Mediated Causes. Sinus bradycardia and AV block can occur in the presence of normal SA and AV nodal 


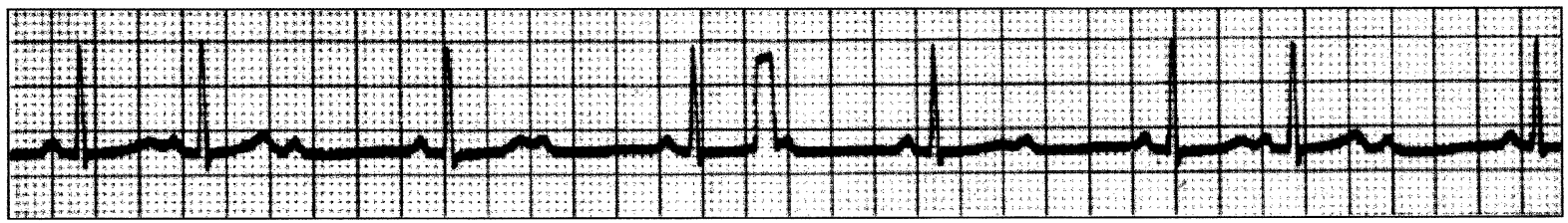

\section{$\mathrm{V}_{1}$}

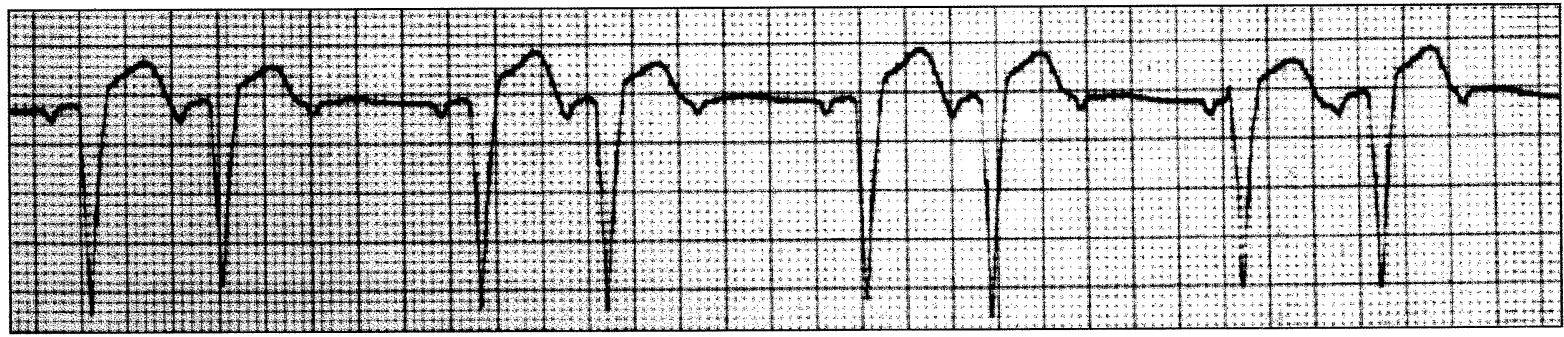

Figure 5. Mobitz II second-degree atrioventricular block develops without P-R interval prolongation. Block occurs below the atrioventricular node. Top, the narrow QRS complexes suggest an intra-His site of block. Bottom, wide QRS complexes are compatible with an infra-His site of block. I, $V$, standard electrocardiographic leads. Adapted with permission from Chou T, Knilans TK (Eds): Electrocardiography in Clinical Practice: Adult and Pediatric. Philadelphia, WB Saunders, 1996, p 453-4.

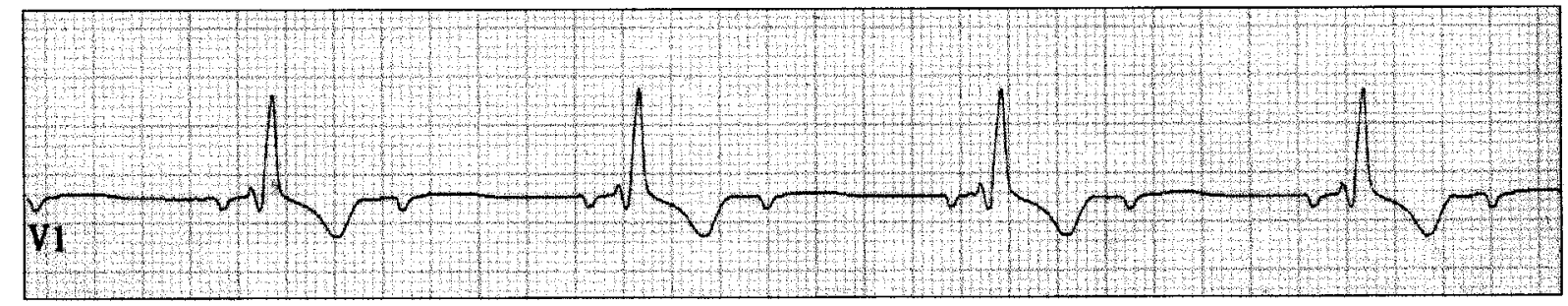

Figure 6. During 2:1 atrioventricular conduction the location of the site of block cannot be determined with certainty from the surface electrocardiogram. This patient's wide QRS complexes suggest block below the bundle of His. $V_{1}$, standard electrocardiographic lead.

function secondary to autonomic instability. Such neurally mediated causes of bradycardia include carotid sinus hypersensitivity and neurocardiogenic (vasovagal) syncope. Although carotid sinus hypersensitivity and typical vasovagal syncope are relatively uncommon in the critical care setting, vagally medicated bradycardia may be very common (Table 2 ). These bradyarrhythmias may be because of several types of stimuli resulting in increased vagal output. The ECG characteristically shows sinus slowing (which is often irregular) with or without $\mathrm{AV}$ block. Typically, no treatment is required because of the transient nature; however, if necessary, these bradyarrhythmias respond to atropine.

Diagnostic Work-Up/Evaluation. Bradyarrhythmias can be caused by either intrinsic disease of the conduction sys- tem or by extrinsic factors acting on the conduction system. In the critical care setting, extrinsic causes are responsible for the majority of bradyarrhythmias. Clinical attention should focus on identifying and correcting reversible causes. Medications, electrolyte disturbances, and increased vagal tone are common causes (Tables 3 and 4).

Documenting a relationship between symptoms and the observed bradycardia is less problematic in the critical care setting (compared with the ambulatory setting) given the universal use of telemetry monitoring.

Electrophysiologic testing may be indicated to determine the site of AV block if further information is needed for clinical decision making. Tilt table and/or electrophysiologic studies may be required if symptoms of presyncope/syncope are thought to be secondary to bradycardia and clinical documentation is unavailable.

Bradyarrhythmias Associated with Acute Myocardial Infarction. Bradycardia is observed in $4 \%$ to $5 \%$ of patients presenting with acute MI (1), and therefore warrants a more detailed discussion. An acute MI can cause bradyarrhythmias by several mechanisms. Sinus node dysfunction may manifest as sinus bradycardia, sinus pauses, or sinus arrest and is most common with inferior MI. The mechanism can be either a direct affect on the SA node blood supply (from the right coronary artery), or stimulation of certain receptors in the inferior left ventricle resulting in increased vagal tone (BezoldJarisch reflex).

Varying degrees of AV block can also be seen in the setting of an acute MI. 
Prognosis and management depend on the location of the MI, degree of AV block, and hemodynamic stability. During inferior infarction, the site of block is known to be in the AV node because the mechanism of AV block is primarily disruption of blood supply to the AV nodal artery, a branch of the RCA. First-degree, second- degree (Mobitz Type 1), and even thirddegree AV block are usually transient and asymptomatic. However, they are also associated with more ventricular tachycardia/ ventricular fibrillation, more hypotension, and a higher in-hospital mortality (5). AV block caused by an anterior MI usually occurs below the AV node; the

Table 1. Distinguishing the site of 2:1 atrioventricular (AV) block

\begin{tabular}{|c|c|c|}
\hline & AV Node & Infranodal \\
\hline Vagal maneuvers/Beta-blockers & Decreased conduction & No change in conduction \\
\hline Atropine & Increased conduction & No change or worsening of AV block \\
\hline Isoproterenol & Increased conduction & May increase conduction \\
\hline
\end{tabular}

area is dependent on blood supply from the LAD. Infranodal block results in second-degree (Mobitz Type II) or thirddegree AV block that is often symptomatic and irreversible.

Medical Treatment. Medical therapy has a limited role in the management of bradyarrhythmias. It is useful in emergent situations (requiring immediate intervention) until pacing can be initiated. Atropine and isoproterenol are the most commonly used medications. Both drugs are useful in increasing the activity of the sinus node and improving block within the AV node. Isoproterenol may also improve infranodal block. Isoproterenol should be used with caution in patients
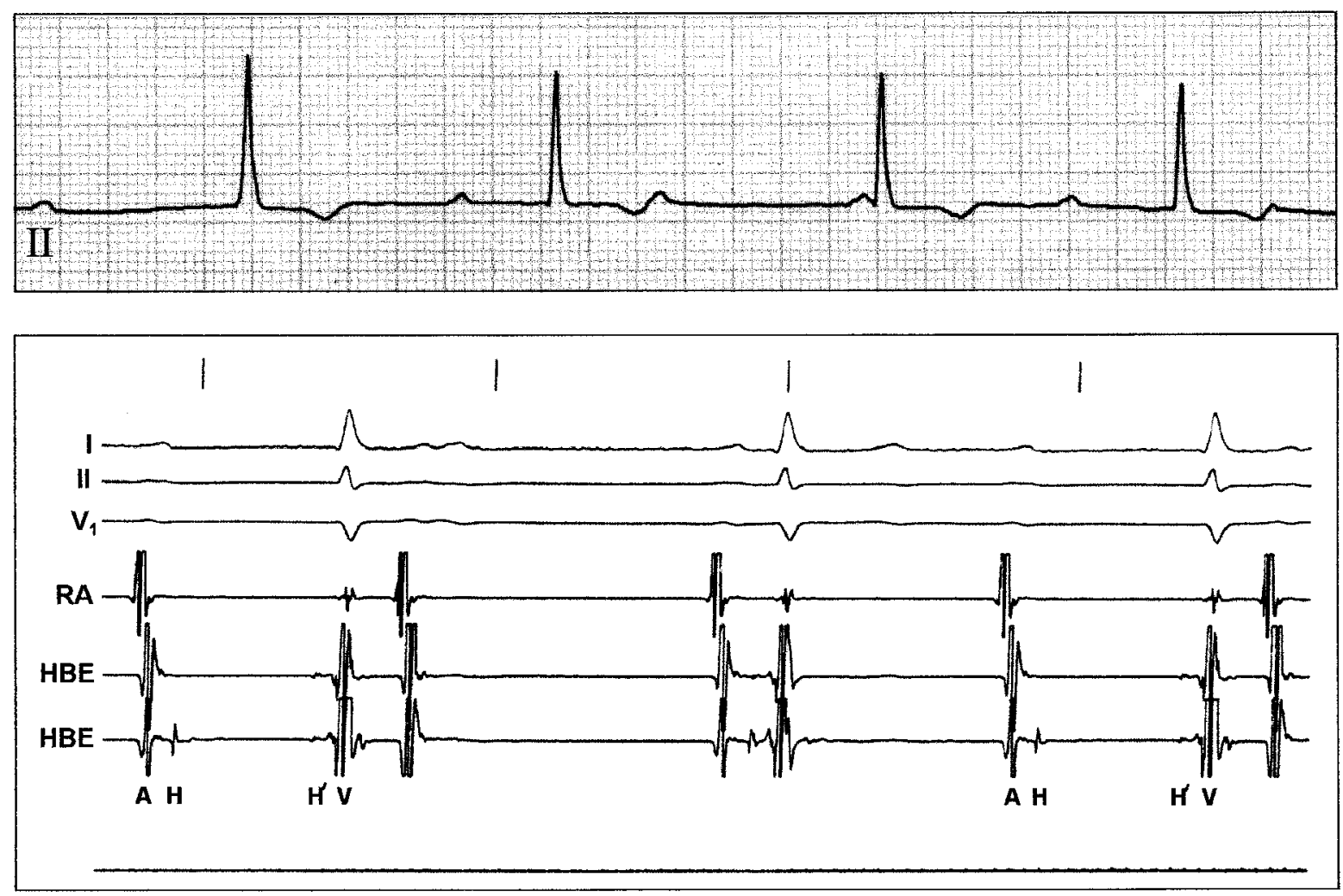

$\mathrm{V}_{1}$

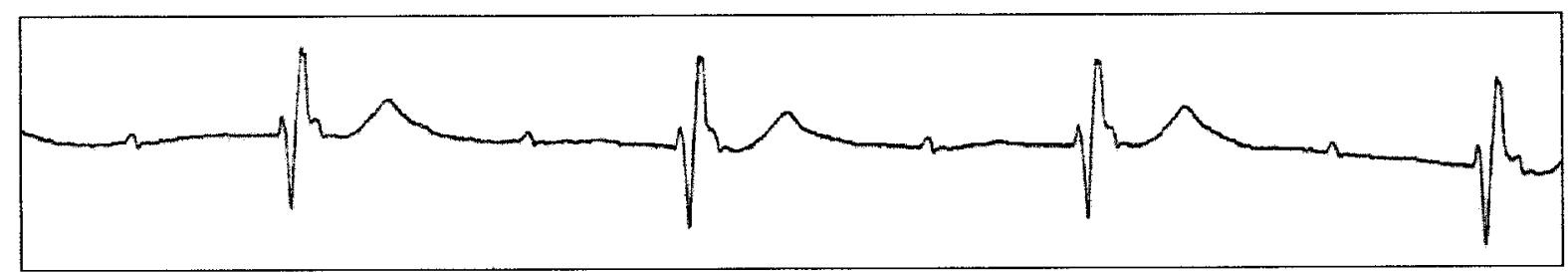

Figure 7. Complete atrioventricular block. Top, narrow QRS complexes suggest an atrioventricular nodal or intra-His block location. Electrophysiologic testing with His bundle recordings may be required to differentiate these entities. Middle, split His $\left(\mathrm{H}\right.$ and $\left.\mathrm{H}^{\prime}\right)$ potentials demonstrate an intra His site of block. RA, HBE, right atrial and His bundle intracardiac electrograms. A, H, H', V, right atrial, proximal His bundle, distal His bundle, and right ventricular intracardiac signals. Bottom, wide QRS complexes again suggest block below the bundle of His. Adapted with permission (4). 
Table 2. Common vagal stimuli encountered in the critical care setting

Intubation
Suctioning
Increased intracranial pressure
Markedly increased blood pressure
Urination
Defecation
Vomiting, retching
Sleep

Table 3. Intrinsic causes of conduction system disease

Degenerative-idiopathic

Coronary artery disease

Cardiomyopathy

Hypertensive heart disease

Infiltrative cardiac diseases (amyloid, sarcoid, neoplasms)

Collagen vascular diseases

Surgical trauma

Myocarditis

Table 4. Extrinsic causes of conduction system disease

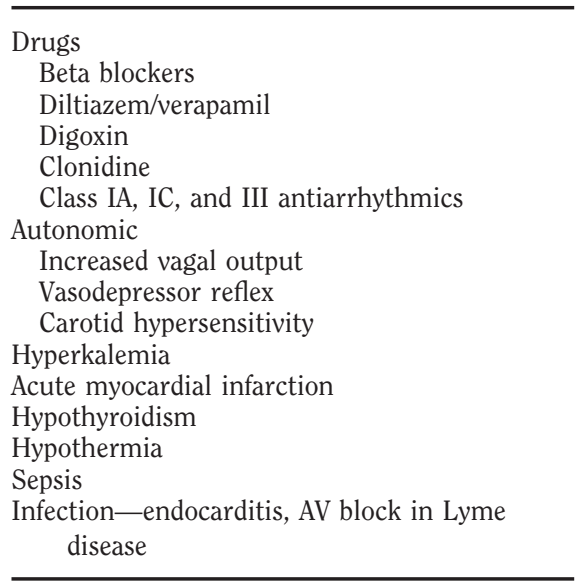

$\mathrm{AV}$, atrioventricular.

with ischemic heart disease. Theophylline may provide modest improvement in patients with mild sinus node dysfunction. Theophylline does not prevent syncope. The availability of small, sophisticated, modern pacemakers makes theophylline's role very limited.

Pacemakers. Pacemakers are the mainstay of therapy for clinically significant bradyarrhythmias. In general, the primary indication for pacing is symptomatic bradycardia causing symptoms of central nervous system hypoperfusion (such as presyncope, syncope, confusion) or hemodynamic compromise. Temporary pacemakers are available for emergent use to stabilize patients with symp-
Table 5. Levels of recommendations by the ACC/AHA task force guidelines

\begin{tabular}{ll}
\hline Class I & $\begin{array}{c}\text { Conditions for which there is evidence or general agreement that pacing is } \\
\text { beneficial. } \\
\text { Conditions for which there is conflicting evidence or divergence of opinion about } \\
\text { the usefulness/effectiveness of pacing } \\
\text { Conditions for which there is evidence and/or general agreement that pacing is not } \\
\text { effective and may be harmful. }\end{array}$ \\
Class III
\end{tabular}

ACC/AHA, American College of Cardiology/American Heart Association.

Adapted with permission (13).

Table 6. ACC/AHA indications for pacing in sinus node dysfunction

Class I 1. Sinus node dysfunction with documented symptomatic bradycardia including frequent sinus pauses that produce symptoms.

2. Symptomatic chronotropic incompetence.

Class II 1. Sinus node dysfunction occurring spontaneously or as a result of necessary drug therapy, with HR $<40$ when a clear association between significant symptoms consistent with bradycardia and the actual bradycardia has not been documented.

2. In minimally symptomatic patients, chronic HR $<30$ while awake.

Class III 1. Sinus node dysfunction in asymptomatic patients including those with substantial bradycardia $(\mathrm{HR}<40)$ a consequence of long-term drug treatment.

2. Sinus node dysfunction in patients with symptoms suggestive of bradycardia that are clearly documented as not associated with a slow heart rate.

3. Sinus node dysfunction with symptomatic bradycardia as a result of nonessential drug therapy.

ACC/AHA, American College of Cardiology/American Heart Association.

Adapted with permission (13).

Table 7. ACC/AHA indications for pacing in acquired atrioventricular (AV) block

Class I 1. Third-degree AV block at any anatomic level, associated with any of the following:

a. Bradycardia with symptoms attributable to AV block.

b. Arrhythmias and other medical conditions that require drugs that result in symptomatic bradycardia.

c. Documented periods of asystole $\geq 3.0$ secs or any escape rate $<40 \mathrm{bpm}$ in awake, symptom-free patients.

d. After-catheter ablation of the AV junction.

e. Postoperative AV block that is not expected to resolve.

f. Neuromuscular diseases with AV block.

2. Second-degree AV block, regardless of type or site of block, that is symptomatic.

Class II 1. Asymptomatic third-degree AV block at any anatomic site level with heart rate $>40 \mathrm{bpm}$.

2. Asymptomatic Type II second-degree heart block.

3. Asymptomatic Type I second-degree AV block at intra- or infra-His levels found incidentally during electrophysiological study for other indications.

4. First-degree AV block with symptoms suggestive of pacemaker syndrome and documented alleviation of symptoms with temporary AV pacing.

5. Marked first-degree AV block ( $>0.30$ secs) in patients with left ventricular dysfunction and symptoms of congestive heart failure in whom a shorter AV interval results in hemodynamic improvement.

Class III 1. Asymptomatic first-degree AV block.

2. Asymptomatic Type I second-degree AV block at the AV node level.

3. AV block expected to resolve and unlikely to recur.

ACC/AHA, American College of Cardiology/American Heart Association; bpm, beats per minute. Adapted with permission (13).

tomatic bradycardia as a bridge to permanent pacing, and for situations where the dysrhythmia is transient or reversible until the underlying cause can be corrected.

Transcutaneous Pacing. Transcutaneous pacing, first introduced by Zoll (6) in 1952 is the oldest form of pacing. Pacing is achieved by stimulation through electrodes placed on the chest wall. Limitations of this procedure include pain with impulse delivery, skeletal muscle stimulation, and decreased ability to capture the ventricle compared with other methods. The role of transcutaneous pacing is currently limited to emergency situations 
Table 8. ACC/AHA indications for permanent pacing after acute myocardial infarction
Class I
1. Persistent second degree AV block in the His-Purkinje system with bilateral bundle-branch block or third-degree AV block within or below the His-Purkinje system after acute myocardial infarction.
2. Transient advanced (second- or third-degree) infranodal AV block and associated bundle-branch block.
3. Persistent and symptomatic second- or third-degree AV block.
Class II 1. Persistent asymptomatic second- or third-degree block at the AV node level.
Class III 1. Transient AV block in the absence of intraventricular conduction defects.
2. Transient AV block in the presence of isolated left anterior fascicular block.
3. Acquired left anterior fascicular block in the absence of AV block.
4. Persistent first-degree AV block in the presence of bundle-branch block of old or indeterminate age.

ACC/AHA, American College of Cardiology/American Heart Association; AV, atrioventricular. Adapted with permission (13).

Table 9. ACC/AHA indications for pacing in chronic bifascicular and trifascicular block
Class I
1. Intermittent third-degree AV block.
2. Type II second-degree heart block.
Class II 1. Syncope not proved to be a result of AV block when other likely causes have been excluded, specifically ventricular tachycardia.
2. Incidental finding at EP testing of markedly prolonged HV interval ( $\geq 100$ msecs) in asymptomatic people.
3. Incidental finding at EP testing of pacing induced infra-His block that is not physiological.
Class III 1. Fascicular block without AV block or symptoms.
2. Fascicular block with first-degree AV block without symptoms.

ACC/AHA, American College of Cardiology/American Heart Association; AV, atrioventricular; EP, electrophysiologic; HV, His-ventricular conduction time measured in msecs.

Adapted with permission (13).

Table 10. ACC/AHA indications for pacing in hypersensitive carotid sinus syndrome and neurally mediated syndromes

\begin{tabular}{|c|c|}
\hline lass I & $\begin{array}{l}\text { 1. Recurrent syncope caused by carotid sinus stimulation or minimal carotid sinus } \\
\text { pressure induces ventricular asystole }>3 \text { secs in absence of culprit medications } \\
\text { which depress the sinus node or AV conduction. }\end{array}$ \\
\hline Class II & $\begin{array}{l}\text { 1. Recurrent syncope without clear, provocative events and with a hypersensitive } \\
\text { cardioinhibitory response. } \\
\text { 2. Syncope of unexplained origin when major abnormalities of sinus node function or } \\
\text { AV conduction are discovered or provoked by EP testing. } \\
\text { 3. Neurally mediated syncope with significant bradycardia reproduced by head-up tilt } \\
\text { testing or other provocative maneuvers. }\end{array}$ \\
\hline I & $\begin{array}{l}\text { 1. A hyperactive cardioinhibitory response to carotid stimulation in the absence of } \\
\text { symptoms. } \\
\text { 2. A hyperactive cardioinhibitory response to carotid stimulation in presence of vague } \\
\text { symptoms. } \\
\text { 3. Recurrent syncope, light-headedness, or dizziness in the absence of a hyperactive } \\
\text { cardioinhibitory response. } \\
\text { 4. Situational vasovagal syncope in which avoidance behavior is effective. }\end{array}$ \\
\hline
\end{tabular}

ACC/AHA, American College of Cardiology/American Heart Association; AV, atrioventricular; EP, electrophysiologic.

Adapted with permission (13).

requiring rapid initiation of pacing (cardiac arrest attributable to bradycardia or asystole as incorporated in the current ACLS protocol).

Epicardial Pacing. Epicardial pacing is frequently encountered in the critical care setting after cardiac surgery. Typically, leads are placed on the epicardium
Recording atrial activity with the epicardial wires during supraventricular tachycardia assists in diagnosing the precise arrhythmia mechanism and helps guide therapy. Ventriculoatrial dissociation or block recorded during wide QRS tachycardia confirms that it is ventricular in origin. Epicardial wires can also be used therapeutically to treat or terminate certain atrial and ventricular tachyarrhythmias by overdrive pacing as well as improving hemodynamic status in selected patients by increasing heart rate (and cardiac output) with pacing.

Transvenous Pacing. Transvenous pacing is the current method of choice for temporary pacing. A pacing catheter is inserted into the heart, directly stimulating the right ventricular myocardium. A percutaneous entry site is chosen, typically either the right internal jugular vein or left subclavian vein as a result of their direct route to the heart. Pacing catheters may be balloon-tipped for easy floatation to the desired site. The usual desired site of pacing is apex of the right ventricle. Special J-shaped wires are also available to allow atrial pacing. If permanent pacing is anticipated, the side opposite the planned permanent pacemaker site should be used to prevent venous thrombosis and reduce the risk of infection of subsequently implanted permanent pacing leads.

Advantages of transvenous pacing include patient comfort and relative ease, speed, and safety of insertion by experienced personnel. Complications have been reported in $4 \%$ to $20 \%$ of patients and are usually observed during prolonged use. These include pneumothorax, myocardial perforation, infection, bleeding, ventricular ectopy/nonsustained ventricular tachycardia, or (rarely) thromboembolism resulting in a pulmonary embolism. A prosthetic tricuspid valve is a contraindication to placement of a transvenous pacer.

Permanent Pacemakers. Permanent pacemakers are placed for persistent, nonreversible symptomatic bradycardia. These pacemakers are placed transvenously, usually via the left subclavian or cephalic vein. Dual-chamber pacemakers (allowing preservation of AV synchrony) with rate-adaptive features, are preferred over single-chamber devices.

In sinus node dysfunction, dualchamber or atrial pacing is preferred over ventricular pacing (7). Data from Benditt et al. (8) in 1990 suggested atrial or dual chamber pacing as the preferred methods 
of pacing, thereby decreasing the incidence of developing atrial fibrillation and thromboembolic events.

Sgarbossa and colleagues (9) subsequently studied the development of chronic atrial fibrillation and stroke in 507 paced patients with sick sinus syndrome. Dual-chamber or atrial pacemakers were used in 395 patients. Stroke and chronic atrial fibrillation were strongly determined by clinical variables and secondarily by a ventricular pacing modality. Ventricular pacing predicted chronic atrial fibrillation only in patients with preimplant paroxysmal atrial fibrillation.

They also found that single-chamber pacing increased the risk of both cardiovascular and total mortality; however, the difference was of borderline statistical significance (10). Ventricular pacing was not associated with an increased incidence of congestive heart failure (11).

A more recent multicenter trial compared quality-of-life benefits in ventricular and dual-chamber pacing. Patients with sinus node dysfunction, but not those with AV block, had moderately better cardiovascular functional status and quality of life with dual-chamber pacing (12).

Atrial demand pacing is adequate when there is no other evidence of $\mathrm{AV}$ nodal conduction disease (1), whereas ventricular demand pacing is indicated in patients with chronic atrial fibrillation with a normal chronotropic response and infrequent bradycardic episodes (1). Dualchamber pacemakers are still preferred in paroxysmal atrial fibrillation. By programming mode switching within these devices, an automatic shift from DDD to DDI or VVI (nontracking) modes during supraventricular arrhythmias helps to avoid ventricular tracking and pacing of rapid atrial rates.

Indications for Pacing. The American College of Cardiology/American Heart Association task force has presented a set of guidelines outlining indications for permanent cardiac pacing. Their recommendations are organized into classes based on clinical and investigational data (Table 5). Tables 6 through 10 contain a selected partial list of the task force recommendations (13).
There are no clinical data to support the efficacy of permanent pacing in patients with asymptomatic sinus node dysfunction (14-16). In general, pacing in sinus node dysfunction is recommended in patients when symptoms can be attributed to bradycardia (Table 6). Indications for pacing in acquired AV block are outlined in Table 7.

As discussed previously, both sinus node dysfunction and AV block can occur in the acute MI setting. Pacing is rarely needed in sinus node dysfunction related to ischemia or infarct. If pacing is required for symptomatic bradycardia, temporary pacing is preferred, as most cases will resolve over time.

In cases of ischemia or infarction resulting in AV block, the level of block is determined by the location of the MI. Temporary pacing is preferred for inferior MI with symptomatic bradycardia, as most conduction disturbances are transient. Permanent pacing is very rarely needed. Acute anterior MI complicated by symptomatic AV block may require temporary pacing for initial stabilization and commonly requires permanent pacing (see Table 8 for pacing indications after acute MI).

Indications for pacing in chronic bifascicular and trifascicular block are outlined in Table 9. Pacing for hypersensitive carotid sinus syndrome and neurally mediated syndromes is outlined in Table 10.

\section{REFERENCES}

1. Prystowsky EN, Klein GJ: Cardiac Arrhythmias: An Approach for the Clinician, New York, McGraw-Hill, 1994, pp 211-223

2. Zipes DP, Miyazaki T: The autonomic nervous system and the heart: Basis for understanding interactions and effects on arrhythmia development. In: Zipes DP, Jalife J (Eds). Cardiac Electrophysiology: From Cell to Bedside. Philadelphia, WB Saunders, 1990, pp 312-330

3. Brodsky M, Wu D, Denes P, et al: Arrhythmias documented by 24 hour continuous electrocardiographic monitoring in 50 male medical students without apparent heart disease. Am J Cardiol 1997; 3:390-395

4. Trohman RG, Donadini M, Palomo AR, et al: Exercise tolerance testing in symptomatic and asymptomatic complete intra-His block. Am Heart J 1987; 13:1252-1254
5. Rardon DP, Miles WM, Zipes DP: Atrioventricular block and dissociation. In: Zipes DP, Jalife J (Eds). Cardiac Electrophysiology: From Cell to Bedside. Philadelphia, WB Saunders, 2000, pp 451-458

6. Zoll PM: Resuscitation of the heart in ventricular standstill by external electric stimulation. $N$ Engl J Med 1952; 247:768-781

7. Robles de Medina EO, Wilde AAM: Sinus bradycardia, sinus arrest, and sinoatrial exit block: Pathophysiological, electrocardiographic, and clinical considerations. In: Zipes DP, Jalife J (Eds). Cardiac Electrophysiology: From Cell to Bedside. Philadelphia, WB Saunders, 2000, pp 447-451

8. Benditt DG, Milstein S, Goldstein M, et al: Sinus node dysfunction: Pathophysiology, clinical features, evaluation and treatment. In: Zipes DP, Jalife J (Eds). Cardiac Electrophysiology: From Cell to Bedside. Philadelphia, WB Saunders, 1990, pp 708-734

9. Sgarbossa EB, Pinski SL, Maloney JD, et al: Chronic atrial fibrillation and stroke in paced patients with sick sinus syndrome: Relevance of clinical characteristics and pacing modalities. Circulation 1993; 88:1045-1053

10. Sgarbossa EB, Pinski SL, Maloney JD: The role of pacing modality in determining longterm survival in the sick sinus syndrome. Ann Intern Med 1993; 119:359-365

11. Sgarbossa EB, Pinski SL, Trohman RG, et al: Single chamber ventricular pacing is not associated with worsening heart failure in sick sinus syndrome. Am J Cardiol 1994; 73: 693-607

12. Lamas GA, Oray EJ, Stambler BS, et al: Quality of life and clinical outcomes in elderly patients treated with ventricular pacing as compared with dual-chamber pacing. Pacemaker Selection in the Elderly Investigators. N Engl J Med 1998; 338:1097-1104

13. Gregoratos G, Cheitlin MD, Gonill A, et al: ACC/AHA guidelines for implantation of cardiac pacemakers and antiarrhythmia devices: Executives summary - a report of the American College of Cardiology/American Heart Association task force on practice guidelines (committee on pacemaker implantation). Circulation 1998; 97:1325-1335

14. Kerr CR, Grant AD, Wenger TL, et al: Sinus node dysfunction. Cardiol Clin 1983; 1:187-207

15. Gann D, Tollentino A, Samet P: Electrophysiologic evaluation of elderly patients with sinus bradycardia: A long-term follow-up study. Ann Intern Med 1979; 90:24-29

16. Mazuz M, Friedman HS: Significance of prolonged electrocardiographic pauses in sinoatrial disease: Sick sinus syndrome. Am J Cardiol 1983; 52:485-489 